\title{
Sulfatase 1 and sulfatase 2 as novel regulators of macrophage antigen presentation and phagocytosis
}

\author{
Hyun-Je Kim ${ }^{1}$, Hee-Sun Kim ${ }^{2}$, Young-Hoon Hong ${ }^{3}$ \\ ${ }^{1}$ Division of Rheumatology, Department of Internal Medicine, CHA University, CHA Gumi Medical Center, Gumi, Korea \\ ${ }^{2}$ Department of Microbiology, Yeungnam University College of Medicine, Daegu, Korea \\ ${ }^{3}$ Division of Rheumatology, Department of Internal Medicine, Yeungnam University College of Medicine, Daegu, Korea
}

Received: March 23, 2021

Revised: May 20, 2021

Accepted: May 25, 2021

Corresponding author: Young-Hoon Hong, MD, PhD Division of Rheumatology, Department of Internal Medicine, Yeungnam University College of Medicine, 170 Hyeonchung-ro, Nam-gu, Daegu 42415, Korea

Tel: +82-53-620-3841

Fax: +82-53-654-8386

E-mail:yhhong@med.yu.ac.kr
Background: Sulfation of heparan sulfate proteoglycans (HSPGs) is critical for the binding and signaling of ligands that mediate inflammation. Extracellular 6-0-endosulfatases regulate posttranslational sulfation levels and patterns of HSPGs. In this study, extracellular 6-0-endosulfatases, sulfatase (Sulf)-1 and Sulf-2, were evaluated for their expression and function in inflammatory cells and tissues.

Methods: Harvested human peripheral blood mononuclear cells were treated with phytohemagglutinin and lipopolysaccharide, and murine peritoneal macrophages were stimulated with interleukin (IL)- $1 \beta$ for the evaluation of Sulf-1 and Sulf-2 expression. Sulf expression in inflammatory cells was examined in the human rheumatoid arthritis (RA) synovium by immunofluorescence staining. The antigen presentation and phagocytic activities of macrophages were compared according to the expression state of Sulfs. Sulfs-knockdown macrophages and Sulfs-overexpressing macrophages were generated using small interfering RNAs and pcDNA3.1 plasmids for Sulf-1 and Sulf-2, respectively.

Results: Lymphocytes and monocytes showed weak Sulf expression, which remained unaffected by IL-1 $\beta$. However, peritoneal macrophages showed increased expression of Sulfs upon stimulation with IL-1 $\beta$. In human RA synovium, two-colored double immunofluorescent staining of Sulfs and CD68 revealed active upregulation of Sulfs in macrophages of inflamed tissues, but not in lymphocytes of lymphoid follicles. Macrophages are professional antigen-presenting cells. The antigen presentation and phagocytic activities of macrophages were dependent on the level of Sulf expression, suppressed in Sulfs-knockdown macrophages, and enhanced in Sulfs-overexpressing macrophages.

Conclusion: The results demonstrate that upregulation of Sulfs in macrophages occurs in response to inflammation, and Sulfs actively regulate the antigen presentation and phagocytic activities of macrophages as novel immune regulators.

Keywords: Antigen presentation; Macrophages; Sulfatase 1; Sulfatase 2

\section{Introduction}

Heparan sulfate proteoglycans (HSPGs) are ubiquitous molecules on the cell surface and matrix in all animal species. They act as re- ceptors, co-receptors, reservoirs, or inhibitors of various ligands, and have many biological implications by binding to growth factors, cytokines, chemokines, adhesion molecules, extracellular matrix components, degradative enzymes, protease inhibitors, and

Copyright (C) 2021 Yeungnam University College of Medicine

This is an Open Access article distributed under the terms of the Creative Commons Attribution Non-Commercial License (http://creativecommons.org/licenses/by-nc/4.0/) which permits unrestricted non-commercial use, distribution, and reproduction in any medium, provided the original work is properly cited. 
proteins involved in lipid metabolism [1].

Accumulating evidence suggests that various proinflammatory cytokines can upregulate the surface expression of specific HSPGs and heparin/heparan sulfates (HS), which participate in inflammatory responses and modulate the interactions with leukocytes [2]. Upon binding to cell surfaces, matrix proteins, and soluble ligands, HSPGs can regulate inflammatory cell maturation and activation, leukocyte rolling, adhesion, extravasation, and chemotaxis [3].

The biological functions of HSPGs can be attributed to the specialized structures within the HS moieties [4]. Posttranslational modifications of HSPGs, such as sulfation, give rise to the molecular diversity and heterogeneity of HSPGs. Cytokines and chemokines bind selectively to the substructures of HSPGs, which determine the specificity of leukocyte recruitment [5-8]. Sulfation of HSPGs is critical for binding and signaling of the ligands that mediate inflammation and is essential for leukocyte rolling and adhesion to regulate inflammation [9]. Antigen-specific B cell differentiation can also be affected by the size of glycosaminoglycans (GAGs) and sulfation of HSPGs [10-12].

The pattern of HSPG sulfation is enzymatically modified by various sulfotransferases and sulfatases (Surfs). Surfs, which are enzymes of the esterase class, catalyze the hydrolysis of sulfate esters and participate in the degradation and modulation of sulfated GAGs in the lysosome [13]. Extracellular 6-O-endosulfatases, recently identified as Sulf- 1 and Sulf- 2 aryl-sulfatases, regulate posttranslational sulfation levels and patterns of HSPGs in extracellular compartments. They selectively remove 6-O-sulfate groups from HSPGs via intramolecular hydrolysis and rearrangement [14-17].

A large amount of data is available regarding HSPGs related to inflammation; however, the effects of modification by sulfation remain unclear. This study was conducted to investigate the cellular expression of Sulfs and their role in inflammation.

\section{Materials and methods}

\section{Reagents}

Easy-blue total RNA extraction kit for total RNA isolation was purchased from iNtRON Biotechnology (Seoul, Korea). Dulbecco's phosphate-buffered saline, penicillin-streptomycin, and fetal bovine serum (FBS) were purchased from Gibco/BRL (Life Technologies, Gaithersburg, MD, USA). Ovalbumin (OVA), phytohemagglutinin (PHA), and lipopolysaccharides (LPS) were obtained from Sigma-Aldrich Co. (St. Louis, MO, USA). Interleukin (IL)- $1 \beta$ and monocyte chemotactic protein-1 (MCP-1) were obtained from R\&D Systems (Minneapolis, MN, USA). The Qiagen Mini kit was purchased from Qiagen Sciences (Germantown, MD, USA). LightCycler FastStart DNA SYBR Green I Mix was obtained from Roche (Mannheim, Germany). Rabbit anti-human Sulf- 1 polyclonal antibody and rabbit anti-human Sulf-2 were purchased from Santa Cruz Biotechnology (CA, USA). Antibodies against CD68 were purchased from Abcam Inc. (Cambridge, MA, USA); goat anti-mouse immunoglobulin $\mathrm{G}(\mathrm{IgG})$ Alexa Fluor 488, goat anti-rabbit IgG Alexa Fluor 546, rabbit anti-goat IgG Alexa Fluor 546, normal goat serum, and normal rabbit serum from Invitrogen Corp. (Carlsbad, CA, USA). Primer sequences for Sulf-1, Sulf-2, and $\beta$-actin were synthesized by Bionics (Daejeon, Korea). Sulf-1 and Sulf-2 small interfering RNA (siRNA) sequences were purchased from Santa Cruz Biotechnology. Negative control siRNA was purchased from Invitrogen (Carlsbad). pcDNA3.1(-) vector for transfection was obtained from Invitrogen Life Technologies (Carlsbad, CA, USA). pcDNA3.1/myc-His(-) Sulf-1 and pcDNA3.1/myc-His(-) Sulf-2 from Addgene Inc. (Cambridge, MA, USA).

\section{Procurement of rheumatoid arthritis synovial tissues and immunofluorescence staining}

With informed consent, synovial tissues were obtained from patients with rheumatoid arthritis (RA) who underwent total knee replacement surgery at Yeungnam University Medical Center. A CD68 primary antibody specific to macrophages and anti-Sulf- 1 , Sulf-2 primary antibodies were applied in parallel for immunofluorescence after pretreatment for paraffin sections and blocking. After overnight incubation with the primary antibodies at $4^{\circ} \mathrm{C}$, the sections were treated with the respective secondary antibodies labeled with fluorescence for 30 minutes avoiding light at room temperature. Cell nuclei were counterstained with Hoechst 33258 diluted at 1:5,000 in distilled water for 2 minutes protected from light at room temperature. Expression of Sulf-1, Sulf-2, and CD68 was observed using a fluorescence microscope, and the images were merged for double staining using Photoshop 7.0 (Adobe Systems Inc., San Jose, CA, USA).

\section{Human peripheral blood mononuclear cells preparation}

Peripheral blood mononuclear cells (PBMCs) were isolated by endotoxin-free Ficoll-Paque PLUS centrifugation (GE Healthcare Bio-Sciences AB, Uppsala, Sweden) from buffy coats obtained from healthy adult blood donors at Yeungnam University Medical Center. PBMCs were cultured at a cell concentration of $10^{6}$ cells/ $\mathrm{mL}$ in RPMI 1640 medium (Lonza, Basel, Switzerland) supplemented with $10 \% \mathrm{FBS}$ and $1 \%$ penicillin-streptomycin. Prior to stimulation, plates were incubated for 1.5 hours $\left(37^{\circ} \mathrm{C}, 5 \% \mathrm{CO}_{2}\right.$, $100 \%$ humidity). 


\section{Mouse peritoneal macrophage preparation}

Specific pathogen-free, 7-week-old inbred BALB/c mice (six mice, three experiments/mouse) were purchased from Central Lab Animal Inc. (Seoul, Korea). The utmost precautions were taken to ensure that the mice remained free from infection by environmental pathogens. The mice were cared for in accordance with the principles of the Guide to the Care and Use of Experimental Animals of Yeungnam Medical Center. Peritoneal macrophages were obtained by lavage using Hanks' balanced salt solution (HBSS) 4 days after injection with $2 \mathrm{~mL}$ of $4 \%$ thioglycollate medium (BBL-Becton Dickinson, Cockeysville, MD, USA). Macrophages in complete medium (RPMI 1640 supplemented with 1\% penicillin-streptomycin and 10\% FBS) were plated in six well tissue culture plates, incubated for 2 hours at $37^{\circ} \mathrm{C}$ in an atmosphere of $5 \% \mathrm{CO}_{2}$, and then washed three times with HBSS to remove any non-adherent cells. Macrophages were cultured overnight in a complete medium at $37^{\circ} \mathrm{C}$ in $5 \% \mathrm{CO}_{2}$. The medium was then replaced with serum-free RPMI 1640, and the cells were cultured in the presence or absence of stimuli for the indicated times.

\section{Sulf-1, Sulf-2 knockdown}

Macrophages were transfected with Sulf-1 and Sulf-2 siRNA oligomers ( 50 nmol/L) using Lipofectamine 2000 (Thermo Fisher Scientific, Waltham, MA, USA) in accordance with the manufacturer's instructions. After 24 hours of incubation, the macrophages were placed in a growth medium for 24 hours before the experiments.

\section{Sulf-1, Sulf-2 overexpression}

pcDNA3.1, pcDNA3.1/Sulf-1, and pcDNA3.1/Sulf-2 plasmids were amplified after transformation into MAX Efficiency DH5 $\alpha$ Competent cells on LB medium with $100 \mu \mathrm{g} / \mathrm{mL}$ ampicillin. The amplified plasmids were purified from cell lysates using the Qiagen Mini kit, according to the manufacturer's instructions. One microgram of each complementary DNA (cDNA) was transfected into macrophages using $2.5 \mu \mathrm{L}$ of Lipofectamine 2000 reagent diluted in Opti-MEM (Thermo Fisher Scientific) separately.

\section{Antigen presentation assay}

Macrophages were cultured with OVA $(2 \mathrm{mg} / \mathrm{mL})$ for 24 hours. T cell hybridomas (DO-11-10, $10^{5}$ cells/well) were added, and the cultures were incubated for 48 hours. The cultures were then centrifuged at $1,000 \times g$, followed by the removal of $100 \mu \mathrm{L}$ of the supernatant. The cell supernatant fluids were assayed for IL-2 activity immediately or frozen at $-70^{\circ} \mathrm{C}$ until use. The supernatant fluids were assayed for the presence of IL- 2 using the IL-2 dependent CTLL- 2 cell line. CTLL- 2 cells $\left(10^{4}\right.$ cells/ well $)$ were added to
$100 \mu \mathrm{L}$ of culture supernatant and incubated for 40 hours. The cultures were then pulsed overnight with $\left[{ }^{3} \mathrm{H}\right]$-thymidine $(1 \mu \mathrm{Ci} /$ well) (Perkin Elmer, Boston, MA, USA). [ $\left.{ }^{3} \mathrm{H}\right]$-Thymidine-labeled cells were collected, and radioactivity was measured using a Packard scintillation counter (Packard Instrument Co., Meriden, CT, USA).

\section{Phagocytosis assay}

Mouse peritoneal macrophages were transfected with siRNA oligomers or control plasmid DNA. After 24 hours of incubation, a phagocytosis assay using a Cytoselect 96-well phagocytosis assay kit was performed according to the manufacturer's instructions (Cell Biolabs, San Diego, CA, USA). Images of phagocytic macrophages were taken with a Leica DFC 495 camera (Leica Microsystems, Cambridge, UK) mounted on a Nikon microscope (Eclipse TE300; Nikon, Tokyo, Japan) at $200 \times$ magnification.

\section{Real-time polymerase chain reaction}

Total RNA was extracted using the easy-BLUE Total RNA Extraction Kit (iNtRON Biotechnology, Seoul, Korea) according to the manufacturer's instructions. One microgram of total RNA per sample was reverse-transcribed using a Maxime RT premix kit (iNtRON Biotechnology, Daejeon, Korea) according to the manufacturer's instructions. cDNA synthesis was performed at $45^{\circ} \mathrm{C}$ for 60 minutes, followed by real-time (RT) inactivation at $95^{\circ} \mathrm{C}$ for 5 minutes. Sulf- 1 and Sulf- 2 were amplified by RT polymerase chain reaction (PCR) using a LightCycler (Roche). The total PCR volume was $20 \mu \mathrm{L}$, and each PCR reaction consisted of LightCycler FastStart DNA SYBR Green I mix (Roche), primer, and $2 \mu \mathrm{L}$ of cDNA. Prior to PCR amplification, the mixture was incubated at $9^{\circ} \mathrm{C}$ for 10 minutes. The amplification step consisted of 45 cycles of denaturation $\left(10\right.$ seconds at $\left.95^{\circ} \mathrm{C}\right)$, annealing ( 5 seconds at the primer-appropriate temperature), and extension ( 10 seconds at $72^{\circ} \mathrm{C}$ ) with fluorescence detection at $72^{\circ} \mathrm{C}$ after each cycle. After the final cycle, melting point analyses of all samples were performed over a temperature range of $65^{\circ} \mathrm{C}-95^{\circ} \mathrm{C}$ with continuous fluorescence detection. $\beta$-Actin was used as a reference gene for the normalization of sample expression levels. The primers used for PCR were as follows: Sulf-1 (195 bp) sense, 5'-tgctgaacagtcaccctgatccaa-3'; antisense, 5'-tcagatgcagggtttggaggttga-3'; Sulf-2 (195 bp) sense, 5'-tcaaagtgacccatcggtgctaca-3', antisense, 5'-agtcacattcttccggtcgcttct-3'; and $\beta$-actin (148 bp) sense, $5^{\prime}$-agagggaaatcgtgcgtgac-3', antisense, 5'-caatagtgatgacctggccgt-3". The mRNA levels of Sulf- 1 and Sulf- 2 were determined by comparing experimental levels to standard curves and were expressed as relative fold expression levels. 


\section{Western blotting}

Total lysates were prepared in PRO-PREP buffer (iNtRON Biotechnology, Seoul, Korea). Protein concentrations were determined by Bradford assay (Bio-Rad, Hercules, CA, USA) using bovine serum albumin as a standard. Twenty micrograms of protein samples were separated by $10 \%$ sodium dodecyl sulfate-polyacrylamide gel electrophoresis and then transferred onto nitrocellulose membranes. Membranes were soaked in 5\% non-fat dried milk in TBST ( $10 \mathrm{mmol} / \mathrm{L}$ Tris/ $\mathrm{HCl} \mathrm{pH} \mathrm{7.5,} 150 \mathrm{mmol} \mathrm{NaCl}$, and $0.05 \%$ Tween-20) for 1 hour, followed by incubation for 16 to 18 hours with primary antibodies against Sulf-1, Sulf-2, and $\beta$-actin at $4^{\circ} \mathrm{C}$. Membranes were then washed three times with TBST for $10 \mathrm{~min}$ utes, followed by incubation with horseradish peroxidase-conjugated secondary antibody for 1 hour at room temperature. Finally, membranes were rinsed three times with TBST for 10 minutes, and antigen-antibody complexes were detected using an enhanced chemiluminescence detection system (LAS-3000; Fujifilm, Tokyo, Japan).

\section{Statistical analyses}

Statistical analysis was performed using SPSS version 12.0 (SPSS Inc., Chicago, IL, USA). Independent sample $t$-test was used for parametric analysis, and Mann-Whitney test was used for nonparametric analysis with a confidence interval of $95 \%$ and $p$-value of $<0.05$.

\section{Results}

HSPGs are involved in signal transduction and may play a role in inflammation. The effects of HSPGs can be modified by the sulfation pattern and level, which is posttranslationally regulated by Sulf- 1 and Sulf- 2 .

In this study, we evaluated Sulf-1 and Sulf-2 expression in inflammatory cells. After harvesting PBMCs from healthy volunteers, the cells were treated with PHA and LPS to activate lymphocytes and monocytes, respectively. Regarding tissue macrophages, murine peritoneal macrophages were stimulated with $\mathrm{IL}-1 \beta$, the key proinflammatory cytokine, and MCP-1, which regulates the migration and infiltration of monocytes and macrophages. Normal non-treated (NT) PBMCs showed weak expression of Sulfs, showing faint bands on western blot (WB), whereas IL-1 $\beta$-stimulated SW1353 showed increased expression of Sulf- 1 and Sulf- 2 as a positive control. Compared with the NT control, human lymphocytes and monocytes showed no change in Sulf expression upon in vitro activation. However, murine peritoneal macrophages showed increased expression of Sulf- 1 and Sulf- 2 following stimulation with IL-1 $\beta$ (Fig. 1A).
The effects of inflammation on the expression of Sulf- 1 and Sulf2 in inflammatory cells were examined in the human RA synovium. RA synovial tissues showed chronic inflammatory reactions of active proliferative villi, hyperplastic fibroblast-like synoviocytes (FLSs) invading sublining tissues, densely infiltrated inflammatory cells forming lymphoid follicles, increased vasculature, thickened interstitium, and fibrosis. Synovial macrophages were recognizable as $\mathrm{CD} 68$ positive cells infiltrated into the sublining layers. Lymphocytes and mononuclear cells were identified as CD68 negative cells of small nuclei clustered in lymphoid follicles. Two-colored double immunofluorescence staining of Sulfs and CD68 was performed to verify the inflammatory cells expressing Sulf- 1 and Sulf2 in RA synovial tissue.

RA synovial tissues were incubated with anti-CD68 antibody, followed by incubation with Alexa Fluor 488 conjugated secondary antibody. CD68 positive cells emitting bright green were detected as tissue macrophages. The synovial tissues were also treated with anti-Sulf- 1 and anti-Sulf- 2 primary antibodies, and Alexa Fluor 546 conjugated secondary antibody demonstrated Sulf- 1 and Sulf-2 emitting orange to red under fluorescence microscopy. Hoechst 33258 was used for counterstaining the nuclei to indicate the cellular expression of the molecules in the synovium.

Sulf-1- and Sulf-2 positive cells were widely distributed throughout the lining and sublining layers of inflamed RA synovium, which included FLSs and macrophages. Merged images revealed synovial macrophages colored bright yellow as a result of dual positive staining of CD68 and Sulfs. Macrophages were densely infiltrated in the sublining layer (Fig. 1B, upper panel). However, lymphoid follicles composed of small lymphocytes were completely negative for both CD68 and Sulfs (Fig. 1B, lower panel).

The results demonstrate that circulating and tissue lymphocytes or monocytes are not reactive for Sulf expression in response to proinflammatory stimuli and inflammation. In contrast, macrophages stimulated and differentiated in inflamed tissues showed active upregulation of Sulf- 1 and Sulf-2.

Based on the Sulf expression of inflammatory cells, this study aimed to evaluate the roles of macrophage Sulf- 1 and Sulf- 2 in inflammation. Macrophages are the prime scavenger and antigen-presenting cells (APC) that participate in the initiation of inflammatory and immune responses. Sulfs-related functional changes in macrophages were analyzed using an antigen presentation assay with the regulation of Sulf expression in murine peritoneal macrophages.

Sulf expression in macrophages was knocked down using siRNAs against Sulf- 1 and Sulf-2 and upregulated by transfection with pcDNA3.1/Sulf-1 and pcDNA3.1/Sulf-2 plasmids. Downregulation of Sulf gene transcription by Sulf- 1 and Sulf-2 siRNAs in mac- 

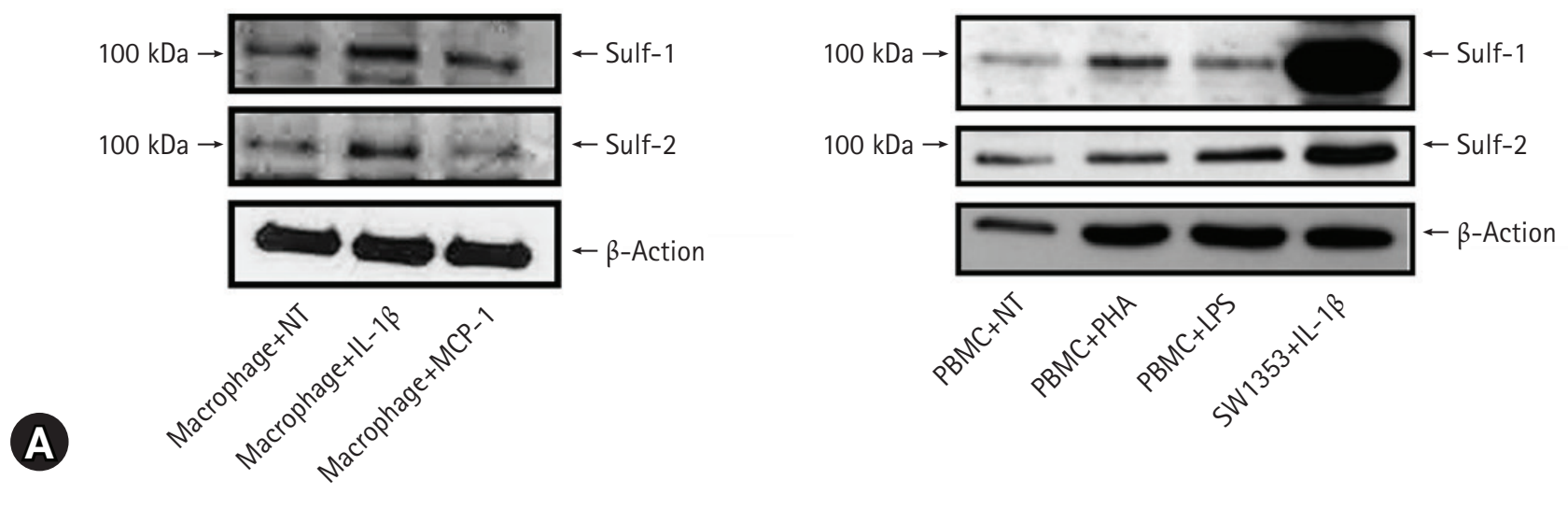

Macrophages in human RA synovium

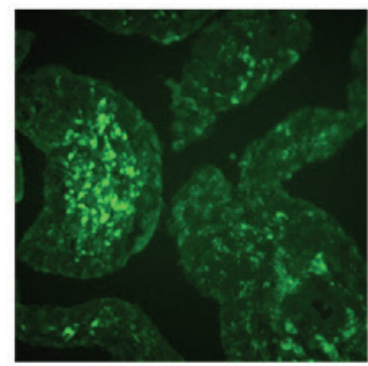

CD68

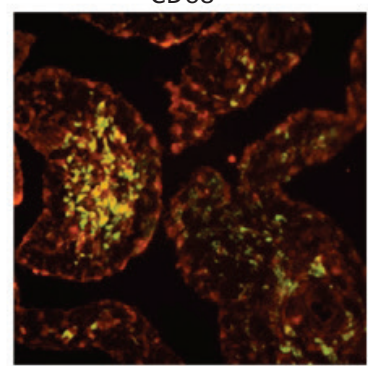

Merge (CD68+Sulf-1)

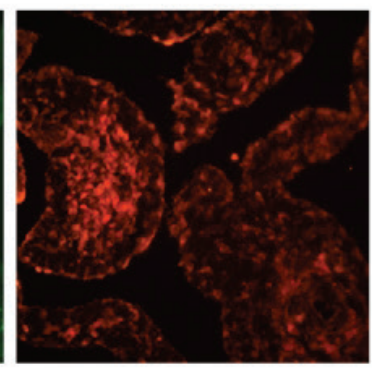

Sulf-1

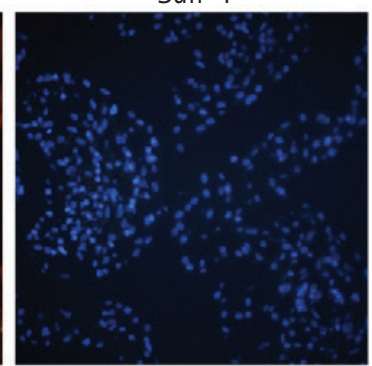

Hoechst

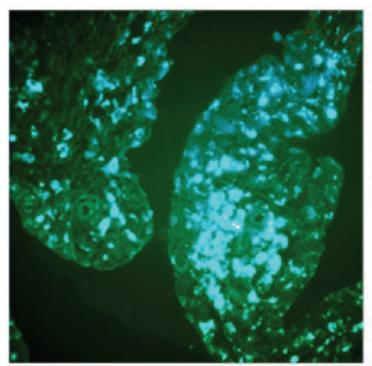

CD68

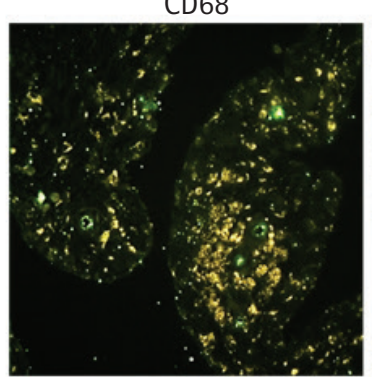

Merge (CD68+Sulf-2)

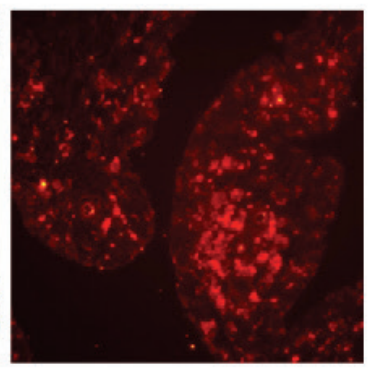

Sulf-2

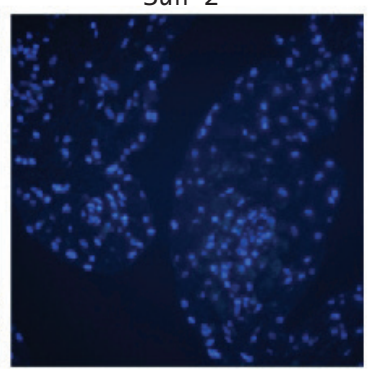

Hoechst

\section{Lymphoid follicles in human RA synovium}

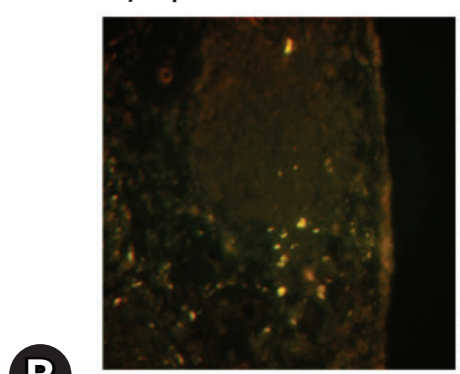

Merge (CD68+Sulf-1)

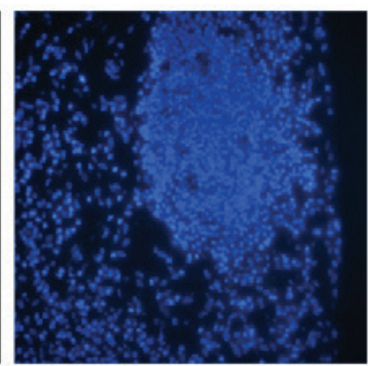

Hoechst

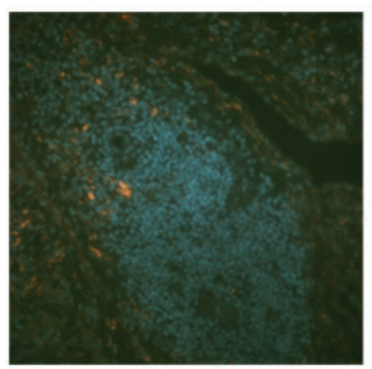

Merge (CD68+Sulf-2)

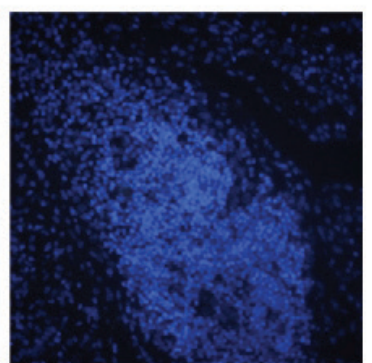

Hoechst

Fig. 1. Sulfatase (Sulf) expression in the inflammatory cells with proinflammatory stimuli and tissue inflammation. Murine peritoneal macrophages show increased expression of Sulf-1 and Sulf-2 upon stimulation with interleukin (IL)-1 $\beta$. (A) However, normal non-treated (NT) peripheral blood mononuclear cells (PBMCs) show weak expression of Sulfs and no change in Sulf expression by in vitro activation with phytohemagglutinin and lipopolysaccharides (LPS) (SW1353, a cell line, as positive control) (two-colored double immunofluorescence stain, x200). (B) Rheumatoid arthritis (RA) synovial tissues show chronic inflammatory reactions. Synovial macrophages as CD68 positive cells infiltrated in sublining layers. Lymphocytes as the CD68 negative cells of small nuclei clustered in lymphoid follicles. (B, upper panel) Two-colored double immunofluorescence staining of Sulfs and CD68 revealed synovial macrophages colored bright yellow as the result of dual positive staining of CD68 and Sulfs. (B, lower panel) However, lymphocytes are completely negative for both CD68 and Sulfs (two-colored double immunofluorescence stain, x200). MCP-1, monocyte chemotactic protein-1. 

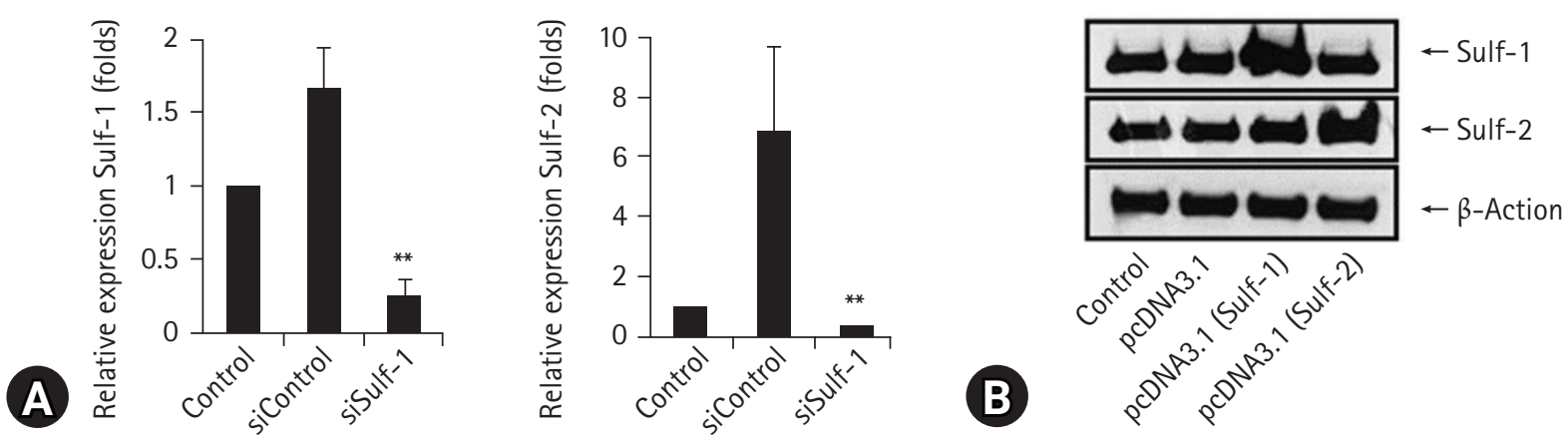

Fig. 2. Macrophage sulfatase (Sulf) expression was knocked down using small interfering (si) RNAs against Sulf-1 and Sulf-2, and upregulated by transfection with pcDNA3.1/Sulf-1 and pcDNA3.1/Sulf-2 plasmids. (A) Downregulation of Sulf gene transcription in macrophages transfected with Sulf-1 and Sulf-2 siRNAs, as compared with non-treated (NT) and siRNAs controls, was confirmed by real-time polymerase chain reaction. (B) Overexpression of Sulf-1 and Sulf-2 in macrophages transfected with pcDNA3.1/Sulf-1 and Sulf-2 plasmids, as compared with those of NT and pcDNA3.1 controls, was confirmed by western blot. ${ }^{* *} p<0.01$.

rophages was confirmed by RT-PCR (Fig. 2A). Overexpression of Sulf- 1 and Sulf- 2 in macrophages by pcDNA3.1/Sulf- 1 and Sulf-2 plasmid transfection was evident on WB (Fig. 2B).

For the antigen presentation assay, murine peritoneal macrophages were cultured with OVA, followed by measurement of $\left[{ }^{3} \mathrm{H}\right]$-thymidine radioactivity incorporated into IL-2 dependent CTLL-2 cells. Macrophages with Sulf-1 or Sulf-2 suppression showed significantly lower $\left[{ }^{3} \mathrm{H}\right]$-thymidine radioactivity compared to controls (control and siControl, respectively). The decrease in radioactivity was more prominent by Sulf- 1 suppression than by Sulf-2 suppression (control: 634,280.6 $\pm 44,596.36$ counts per minute $[\mathrm{cpm}]$, siControl: $656,073.1 \pm 56,295.45 \mathrm{cpm}$, siSulf-1: $408,304.3 \pm 27,941.08 \mathrm{cpm}$, siSulf-2: 531,577.8 $\pm 37,844.28 \mathrm{cpm}$; values are mean \pm standard deviation $[\mathrm{SD}]$, respectively) (Fig. 3A).

The same experiment was performed in macrophages overexpressing Sulf- 1 or Sulf- 2 to confirm the effects of Sulfs on antigen presentation by macrophages. After incubation with OVA, murine peritoneal macrophages were assessed for antigen-presenting function as $\left[{ }^{3} \mathrm{H}\right]$-thymidine radioactivity of IL-2 dependent CTLL-2 cells. Macrophages overexpressing Sulf-1 or Sulf- 2 showed significantly higher $\left[{ }^{3} \mathrm{H}\right]$-thymidine radioactivity than controls (control and pcDNA3.1, respectively). The increase in radioactivity was more prominent by Sulf- 1 overexpression than by Sulf- 2 overexpression (control: 419,426.67 $\pm 7,843.9 \mathrm{cpm}$, pcDNA3.1: 421,824 $\pm 4,545.04$ cpm pcDNA3.1 (Sulf-1): 870,695.67 $\pm 15,042.13$ cpm, pcDNA3.1 (Sulf-2): 664,237.67 $\pm 29,535.9$ cpm; values are mean \pm SD, respectively) (Fig. 3B). The results showed an inverse correlation with the results of the antigen presentation assay with Sulfs-knockdown macrophages.

The antigen presentation studies coherently suggest that Sulfs of macrophages may take part in antigen presentation and play a role in initiating immune and inflammatory responses. These effects were more prominent with Sulf-1.

Antigen presentation by macrophages involves a wide range of responses initiated by pattern recognition and phagocytosis. Therefore, macrophage phagocytic activity was assessed by regulating Sulf- 1 and Sulf- 2 expression in the same manner as that in the antigen presentation assay. Murine peritoneal macrophages were treated with siRNAs or pcDNA3.1 plasmids to suppress or enhance Sulf expression, respectively (Fig. 2).

Macrophages were cultured with zymosan, and the number of zymosan granules engulfed by macrophages was compared using microscopic images. Sulfs-knockdown macrophages showed marked suppression of zymosan phagocytosis compared to controls (Fig. 4A, upper panel). Macrophages overexpressing Sulfs showed brisk phagocytic activity against zymosan (Fig. 4A, lower panel), which was completely opposite to the Sulfs-knockdown macrophages. In addition to the microscopic images, phagocytosis of zymosan by macrophages was analyzed quantitatively by measuring the absorbance at $450 \mathrm{~nm}$ using spectrophotometry. The absorbance of Sulfs-knockdown macrophages was significantly lower than that of controls (zymosan+control and zymosan+siControl, respectively) (control: 1.28 \pm 0.04 absorbance unit [AU], zymosan+cytochalasin D: $1.49 \pm 0.03 \mathrm{AU}$, zymosan+control: $2.23 \pm 0.05 \mathrm{AU}$, zymosan+siControl: $2.07 \pm 0.03 \mathrm{AU}$, zymosan+ Sulf-1 siRNA: $1.6 \pm 0.1$ AU, zymosan+Sulf-2 siRNA: $1.74 \pm 0.09$ $\mathrm{AU}$; values are mean $\pm S D$, respectively). Inversely, Sulfs- overexpressing macrophages showed higher absorbance compared with controls (zymosan+control and zymosan + pcDNA3.1, respectively) (control: $1.28 \pm 0.04 \mathrm{AU}$, zymosan+cytochalasin D: $1.49 \pm 0.03$ $\mathrm{AU}$, zymosan+control: $2.23 \pm 0.05 \mathrm{AU}$, zymosan+pcDNA3.1: $2.19 \pm 0.03 \mathrm{AU}$, zymosan+pcDNA3.1 [Sulf-1]: $2.97 \pm 0.07 \mathrm{AU}$ zymosan+pcDNA3.1 [Sulf-2]: $2.78 \pm 0.08 \mathrm{AU}$; values are mean $\pm \mathrm{SD}$, respectively). The effects of Sulfs on macrophage phagocyto- 

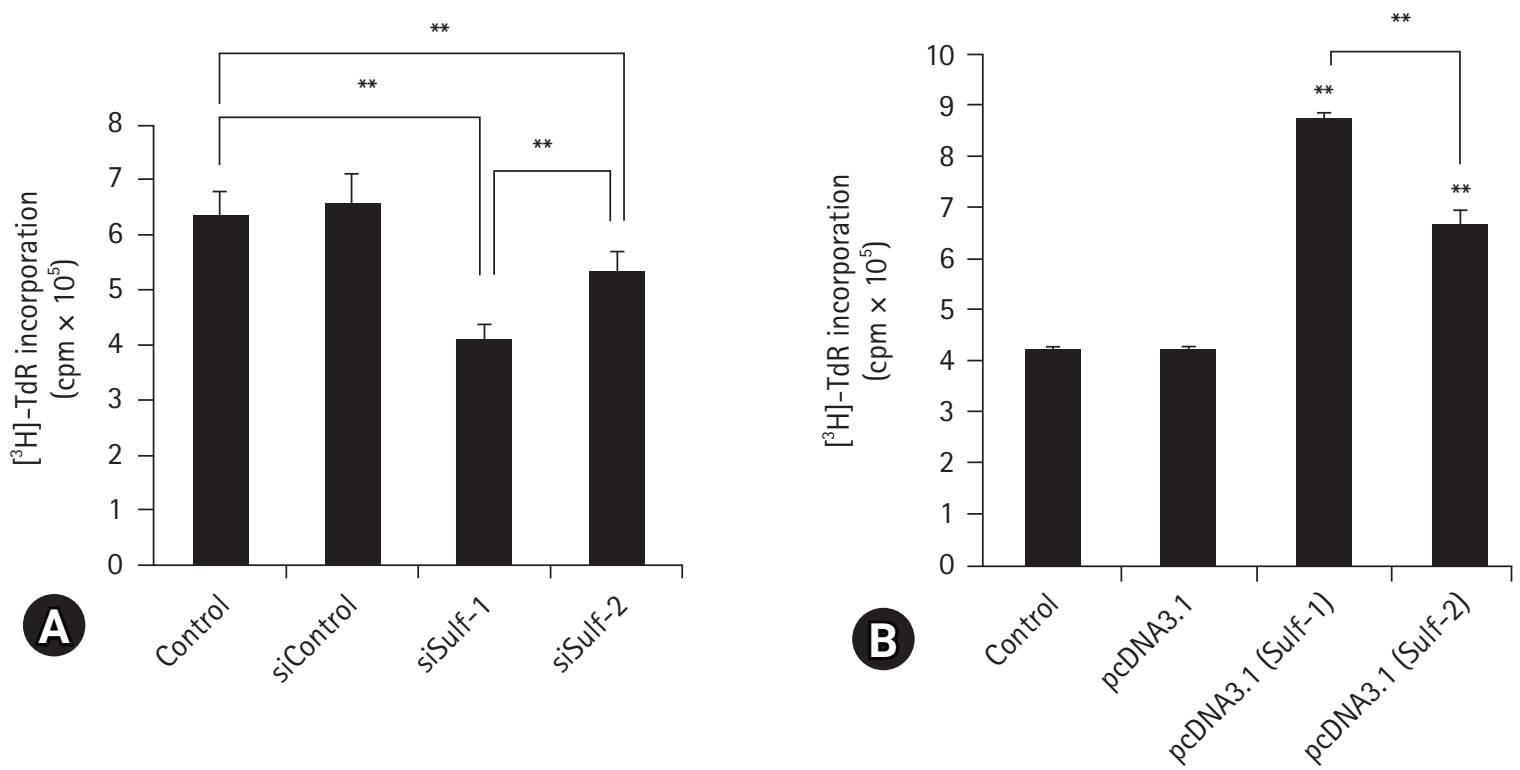

Fig. 3. Antigen presentation assay with modification of sulfatase (Sulf) expression. Murine peritoneal macrophages were cultured with ovalbumin. (A) Macrophages with Sulf-1 or Sulf-2 suppression show significantly lower $\left[{ }^{3} \mathrm{H}\right]$-thymidine radioactivity incorporated into interleukin-2 dependent CTLL-2 cells as compared with controls. (B) Macrophages with upregulation of Sulf-1 or Sulf-2 show significantly higher $\left[{ }^{3} \mathrm{H}\right]$-thymidine radioactivity compared with controls. The $\left[{ }^{3} \mathrm{H}\right]$-thymidine radioactivity is reciprocal by Sulf suppression and overexpression. The changes in radioactivity are more prominent with Sulf-1 regulation than with Sulf-2. cpm, counts per minute. ${ }^{* *} p<0.01$.

sis were more prominent with Sulf-1 compared to Sulf-2 (Fig. 4B).

The results of the macrophage phagocytosis assay with modification of Sulf expression were consistent with the results of Sulfs-regulated antigen presentation by macrophages. This may be evidence of the critical involvement of Sulf- 1 and Sulf- 2 in inflammatory and immune responses regulating macrophage phagocytosis and antigen presentation.

\section{Discussion}

HSPGs are extracellular matrix glycoproteins modified with specific HS polymers [18]. HS is a linear polysaccharide of a variably sulfated repeating disaccharide (GAG). Covalently linked to various core proteins, one or more HS chains comprise extraordinarily heterogeneous structures of HSPGs. The cell-surface HSPG molecules are classified into four groups; syndecans, glypicans, betaglycans, and CD44 family proteins. In addition, the sulfation pattern and level of HSPGs at four different sites (N-, 3-O, and 6-O of glucosamine and 2-O of uronic acid) give rise to molecular diversity [19].

Regarding HSPGs related to infection/inflammation, HSPG is rarely expressed in normal resting leukocytes [20]. However, as monocytes undergo progressive differentiation into tissue macrophages during the recruitment process, activated human macrophages express syndecan-2 on the cell surface [2]. HSPGs are known to facilitate morphogen gradients that are essential for cell development and chemokine gradients for leukocyte recruitment and homing $[21,22]$. In addition to the presence of HSPGs in inflammatory cells, studies on Chlamydia muridarum have demonstrated that the level of HSPG 6-O sulfation is a critical determinant of infection, and that 6-O endosulfatases are involved in microbial pathogenesis as modulators [23].

The sulfation pattern and level of variably sulfated molecules may be the major determinants of the biological activities of HSPGs. Regarding posttranslational modulation, the extracellular 6-O-endosulfatases, Sulf- 1 and Sulf-2, desulfate HSPGs in the extracellular compartment, in contrast to the biosynthesis and sulfation at the Golgi apparatus [24].

Aberrant expression and activity of Sulf- 1 and Sulf- 2 have been linked to diseases such as cancers [25-28] and developmental changes [29-31]. However, little information is available concerning their role in inflammation. Sulf- 1 and Sulf- 2 expression were reported to be upregulated in articular cartilages and synovial tissues of human osteoarthritis (OA) and murine OA knees [32]. These findings might indicate an association between Sulfs and the reactive synovium. Although no information is available about Sulf-1 and Sulf- 2 in inflammatory tissues, the current study shows that synoviocytes and synovial macrophages upregulate Sulf- 1 and Sulf-2 expression in the inflamed synovium of RA.

In this study, Sulf- 1 and Sulf- 2 were analyzed with regard to their 


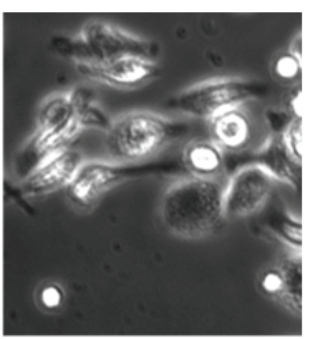

Cont

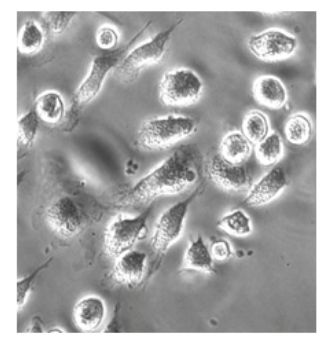

siCont

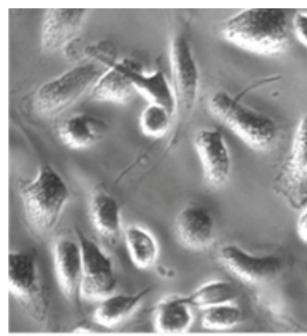

siCont+zymosan

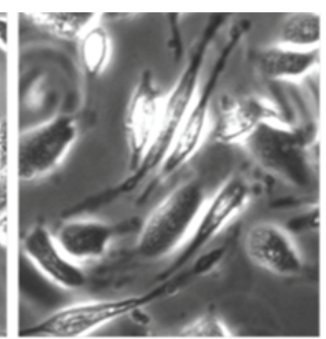

siSulf-1+zymosan

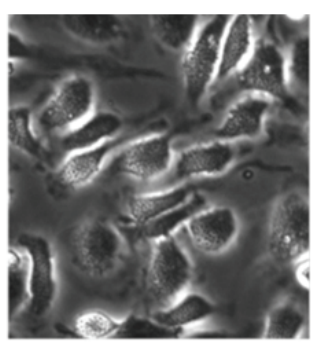

siSulf-2+zymosan

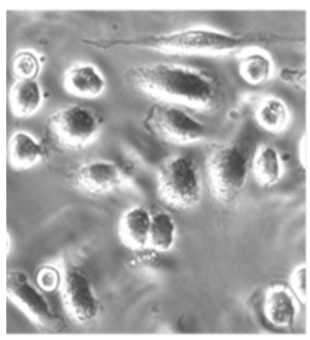

A

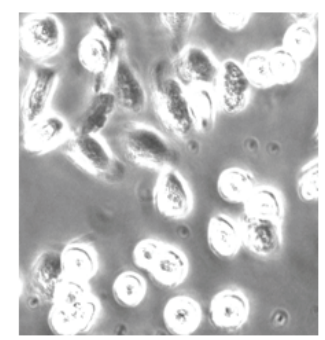

pcDNA3.1

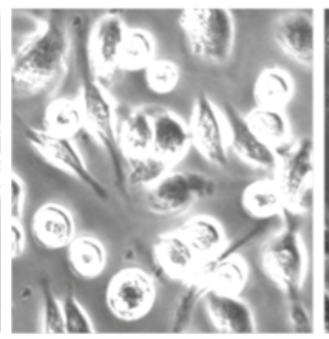

pcDNA3.1+zymosan

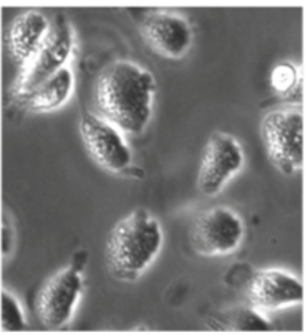

pcDNA3.1sulf-1 +zymosan

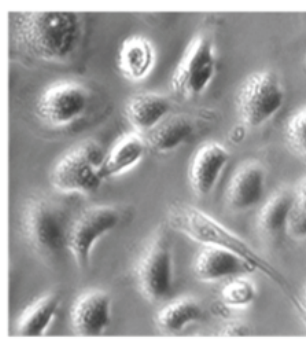

pcDNA3.1sulf-2 +zymosan
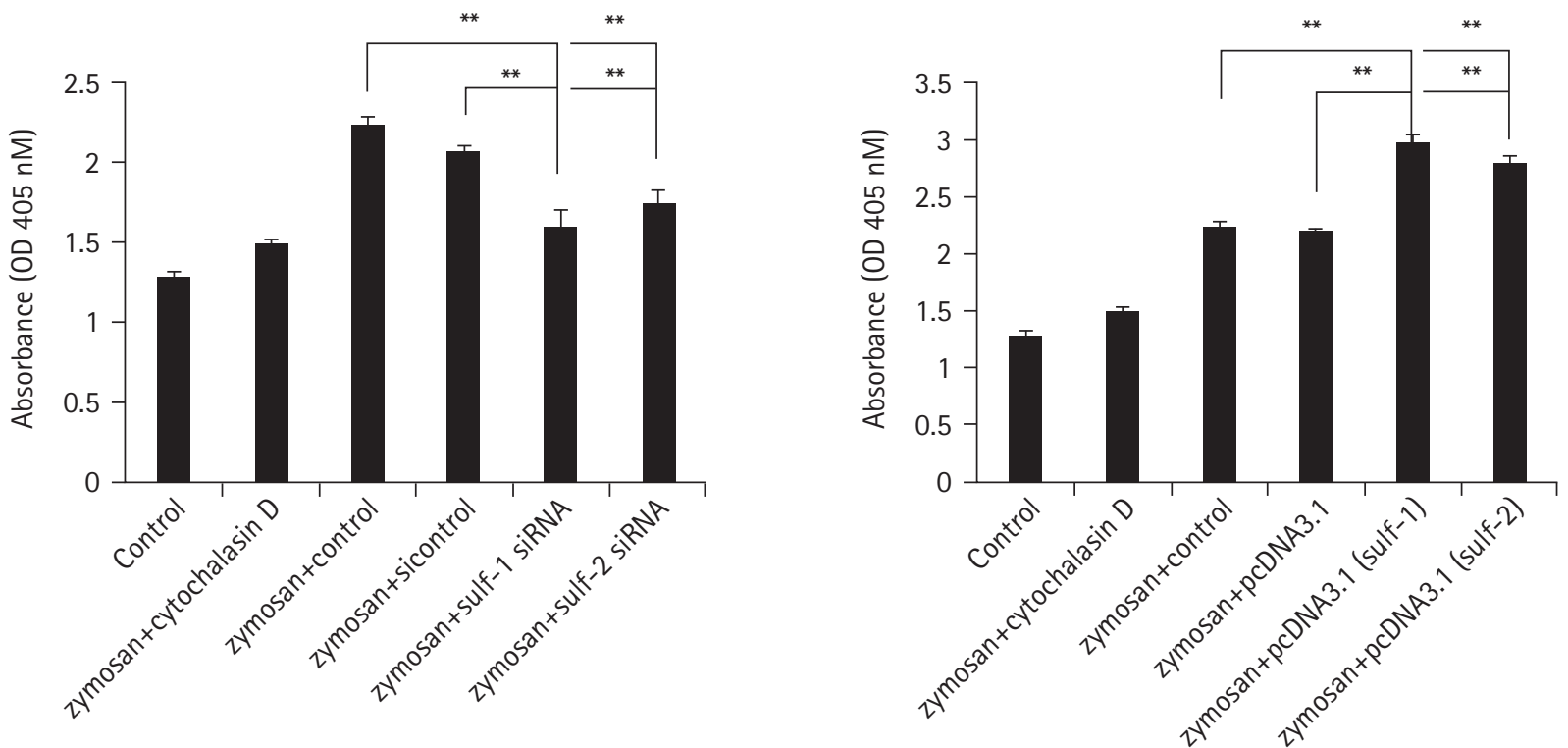

Fig. 4. Macrophage phagocytosis assay with modification of sulfatase (Sulf) expression. Macrophages were cultured with zymosan. (A, upper row) Sulfs-knockdown macrophages show marked suppression of zymosan phagocytosis compared with controls (Cont) on microscopic images. (A, lower row) Sulfs-overexpressing macrophages show brisk phagocytotic activity against zymosan. The phagocytic activity is analyzed by measuring the absorbance at $450 \mathrm{~nm}$ using spectrophotometry. ( $\mathrm{B}$, left) The absorbance of Sulfs-knockdown macrophages was significantly lower than that of controls. (B, right) Sulfs-overexpressing macrophages demonstrated higher absorbance compared with controls. The effects of Sulfs on macrophage phagocytosis are more prominent with Sulf-1 than with Sulf-2. si, small interfering; $0 D$, optical density; $A U$, absorbance unit. ${ }^{* *} p<0.01$.

expression in inflammatory cells of peripheral blood and tissues, and their functions in inflammation.

The results demonstrated that macrophages expressed Sulf- 1 and Sulf-2. In addition, Sulfs are upregulated in macrophages in response to proinflammatory stimuli and tissue inflammation, but not in lymphocytes or monocytes.
Infiltrated macrophages are the core features of inflammation, in which they play roles in eliminating infection, clearing up debris, and restoring tissue homeostasis as an effector of innate immunity [33]. In addition, macrophages, as professional APCs, are involved in the induction of acquired immunity that requires coordinated interplay between innate immune cells and naive lymphocytes. 
The capacity to take up and process antigens, express class II major histocompatibility complex (MHC) glycoproteins on their surface, and synthesize and release IL- 1 are indispensable for the cells to function as APCs [34].

$\mathrm{T}$ cell stimulation by antigen presentation depends on the efficiency of antigen capture by APCs [35], and HSPGs behave as receptors or co-receptors in MHC class II-restricted antigen presentation of proteins [36]. Earlier studies have reported that a critical level of HSPG ligation is necessary and sufficient to trigger phagocytic uptake into epithelial cells [37]. Therefore, in the context of HSPGs and their sulfation as key determinants of biological activities, Sulfs may function as regulators of $\mathrm{T}$ cell activation and immunity by modulating antigen presentation and phagocytosis by APCs.

This study demonstrates that antigen presentation activity by macrophages is strongly correlated with Sulf expression, suppressed in Sulf- 1 and Sulf- 2 knockdown macrophages, and enhanced in Sulfs-overexpressing macrophages.

For the processing and presentation of phagocytosed antigenic substrates to lymphocytes, exogenous antigen uptake into professional APCs is initiated by some types of endocytic mechanisms: receptor-mediated endocytosis through the clathrin-coated pit system [38], pinocytosis, particularly macropinocytosis as a consequence of membrane ruffling [39] and phagocytosis [40].

In relation to the Sulfs-dependent antigen presentation, the current study evaluated macrophage phagocytosis and showed that the phagocytic activity of macrophages also had a strong correlation with Sulf expression, enhanced in Sulf- 1 and Sulf-2 overexpressing macrophages, and vice versa. This is entirely concordant with the results of the antigen presentation assay of macrophages to $\mathrm{T}$ lymphocytes. However, in this study, the protein expression levels were not determined after Sulf-1 and Sulf-2 gene suppression was confirmed by RT-PCR, which could be a limitation of the assay.

Taken together, these observations clearly demonstrate that Sulf1 and Sulf- 2 expression is actively regulated by macrophages in correlation with tissue inflammation, and that Sulfs, particularly Sulf1 , may play a role in facilitating inflammation through macrophage phagocytosis and antigen presentation. To the best of our knowledge, this is the first report on Sulf- 1 and Sulf- 2 as novel immune regulators.

\section{Notes}

\section{Ethical statements}

The study protocol was approved by the Institutional Review Board (IRB) of Yeungnam University Hospital (IRB No: YUH-
12-0305-O2). Informed consent was obtained from participants, which was confirmed by the IRB. This study was conducted in accordance with the Code of Ethics of the World Medical Association (Declaration of Helsinki).

\section{Conflicts of interest}

No potential conflict of interest relevant to this article was reported.

\section{Author contributions}

Conceptualization, Data curation, Formal analysis, Methodology, Project administration, Visualization, Investigation, Resources, Validation: all authors; Software, Supervision: HSK, YHH; Writing original draft: all authors; Writing-review \& editing: all authors.

\section{ORCID}

Hyun-Je Kim, https://orcid.org/0000-0003-1075-5645

Hee Sun Kim, https://orcid.org/0000-0002-2000-4071

Young-Hoon Hong, https://orcid.org/0000-0001-8119-0464

\section{References}

1. Dreyfuss JL, Regatieri CV, Jarrouge TR, Cavalheiro RP, Sampaio LO, Nader HB. Heparan sulfate proteoglycans: structure, protein interactions and cell signaling. An Acad Bras Cienc 2009;81:409-29.

2. Clasper S, Vekemans S, Fiore M, Plebanski M, Wordsworth P, David G, et al. Inducible expression of the cell surface heparan sulfate proteoglycan syndecan-2 (fibroglycan) on human activated macrophages can regulate fibroblast growth factor action. J Biol Chem 1999;274:24113-23.

3. Parish CR. The role of heparan sulphate in inflammation. Nat Rev Immunol 2006;6:633-43.

4. Vanpouille C, Deligny A, Delehedde M, Denys A, Melchior A, Liénard X, et al. The heparin/heparan sulfate sequence that interacts with cyclophilin $\mathrm{B}$ contains a 3-O-sulfated $\mathrm{N}$-unsubstituted glucosamine residue.J Biol Chem 2007;282:24416-29.

5. Matsuo I, Kimura-Yoshida C. Extracellular modulation of fibroblast growth factor signaling through heparan sulfate proteoglycans in mammalian development. Curr Opin Genet Dev 2013; 23:399-407.

6. Witt DP, Lander AD. Differential binding of chemokines to glycosaminoglycan subpopulations. Curr Biol 1994;4:394-400.

7. Wang L, Fuster M, Sriramarao P, Esko JD. Endothelial heparan sulfate deficiency impairs L-selectin- and chemokine-mediated neutrophil trafficking during inflammatory responses. Nat Immunol 2005;6:902-10. 
8. Rops AL, van den Hoven MJ, Baselmans MM, Lensen JF, Wijnhoven TJ, van den Heuvel LP, et al. Heparan sulfate domains on cultured activated glomerular endothelial cells mediate leukocyte trafficking. Kidney Int 2008;73:52-62.

9. Kawashima H. Roles of sulfated glycans in lymphocyte homing. Biol Pharm Bull 2006;29:2343-9.

10. van der Voort R, Keehnen RM, Beuling EA, Spaargaren M, Pals ST. Regulation of cytokine signaling by B cell antigen receptor and CD40-controlled expression of heparan sulfate proteoglycans. J Exp Med 2000;192:1115-24.

11. Floris S, van den Born J, van der Pol SM, Dijkstra CD, De Vries HE. Heparan sulfate proteoglycans modulate monocyte migration across cerebral endothelium. J Neuropathol Exp Neurol 2003;62:780-90.

12. Celie JW, Rutjes NW, Keuning ED, Soininen R, Heljasvaara R, Pihlajaniemi T, et al. Subendothelial heparan sulfate proteoglycans become major L-selectin and monocyte chemoattractant protein-1 ligands upon renal ischemia/reperfusion. Am J Pathol 2007; 170:1865-78.

13. Diez-Roux G, Ballabio A. Sulfatases and human disease. Annu Rev Genomics Hum Genet 2005;6:355-79.

14. Dhoot GK, Gustafsson MK, Ai X, Sun W, Standiford DM, Emerson CP Jr. Regulation of Wnt signaling and embryo patterning by an extracellular sulfatase. Science 2001;293:1663-6.

15. Morimoto-Tomita M, Uchimura K, Werb Z, Hemmerich S, Rosen SD. Cloning and characterization of two extracellular heparin-degrading endosulfatases in mice and humans. J Biol Chem 2002;277:49175-85.

16. Ohto T, Uchida H, Yamazaki H, Keino-Masu K, Matsui A, Masu M. Identification of a novel nonlysosomal sulphatase expressed in the floor plate, choroid plexus and cartilage. Genes Cells 2002;7:173-85.

17. Ai X, Do AT, Lozynska O, Kusche-Gullberg M, Lindahl U, Emerson CP Jr. QSulf1 remodels the 6-O sulfation states of cell surface heparan sulfate proteoglycans to promote Wnt signaling. J Cell Biol 2003;162:341-51.

18. Bernfield M, Götte M, Park PW, Reizes O, Fitzgerald ML, Lincecum J, et al. Functions of cell surface heparan sulfate proteoglycans. Annu Rev Biochem 1999;68:729-77.

19. Esko JD, Lindahl U. Molecular diversity of heparan sulfate. J Clin Invest 2001;108:169-73.

20. Jackson DG. Human leucocyte heparan sulphate proteoglycans and their roles in inflammation. Biochem Soc Trans 1997;25: $220-4$.

21. Camp RL, Scheynius A, Johansson C, Puré E. CD44 is necessary for optimal contact allergic responses but is not required for normal leukocyte extravasation. J Exp Med 1993;178:497-
507.

22. Mikecz K, Brennan FR, Kim JH, Glant TT. Anti-CD44 treatment abrogates tissue oedema and leukocyte infiltration in murine arthritis. Nat Med 1995;1:558-63.

23. Kim JH, Chan C, Elwell C, Singer MS, Dierks T, Lemjabbar-Alaoui H, et al. Endosulfatases SULF1 and SULF2 limit Chlamydia muridarum infection. Cell Microbiol 2013;15: 1560-71.

24. Habuchi H, Habuchi O, Kimata K. Sulfation pattern in glycosaminoglycan: does it have a code? Glycoconj J 2004;21:47-52.

25. Nawroth R, van Zante A, Cervantes S, McManus M, Hebrok M, Rosen SD. Extracellular sulfatases, elements of the Wnt signaling pathway, positively regulate growth and tumorigenicity of human pancreatic cancer cells. PLoS One 2007;2:e392.

26. Lemjabbar-Alaoui H, van Zante A, Singer MS, Xue Q, Wang YQ, Tsay D, et al. Sulf-2, a heparan sulfate endosulfatase, promotes human lung carcinogenesis. Oncogene 2010;29:635-46.

27. Bret C, Moreaux J, Schved JF, Hose D, Klein B. SULFs in human neoplasia: implication as progression and prognosis factors. J Transl Med 2011;9:72.

28. Yang JD, Sun Z, Hu C, Lai J, Dove R, Nakamura I, et al. Sulfatase 1 and sulfatase 2 in hepatocellular carcinoma: associated signaling pathways, tumor phenotypes, and survival. Genes Chromosomes Cancer 2011;50:122-35.

29. Nagamine S, Koike S, Keino-Masu K, Masu M. Expression of a heparan sulfate remodeling enzyme, heparan sulfate 6-O-endosulfatase sulfatase FP2, in the rat nervous system. Brain Res Dev Brain Res 2005; 159:135-43.

30. Buono M, Visigalli I, Bergamasco R, Biffi A, Cosma MP. Sulfatase modifying factor 1-mediated fibroblast growth factor signaling primes hematopoietic multilineage development. J Exp Med 2010;207:1647-60.

31. Langsdorf A, Schumacher V, Shi X, Tran T, Zaia J, Jain S, et al. Expression regulation and function of heparan sulfate 6-O-endosulfatases in the spermatogonial stem cell niche. Glycobiology 2011;21:152-61.

32. Otsuki S, Taniguchi N, Grogan SP, D'Lima D, Kinoshita M, Lotz M. Expression of novel extracellular sulfatases Sulf- 1 and Sulf- 2 in normal and osteoarthritic articular cartilage. Arthritis Res Ther 2008;10:R61.

33. Murray PJ, Wynn TA. Protective and pathogenic functions of macrophage subsets. Nat Rev Immunol 2011;11:723-37.

34. Roche PA, Furuta K. The ins and outs of MHC class II-mediated antigen processing and presentation. Nat Rev Immunol 2015;15:203-16.

35. Guermonprez P, Valladeau J, Zitvogel L, Théry C, Amigorena S. Antigen presentation and $\mathrm{T}$ cell stimulation by dendritic cells. 
Annu Rev Immunol 2002;20:621-67.

36. Léonetti M, Gadzinski A, Moine G. Cell surface heparan sulfate proteoglycans influence MHC class II-restricted antigen presentation. J Immunol 2010;185:3847-56.

37. Dehio C, Freissler E, Lanz C, Gómez-Duarte OG, David G, Meyer TF. Ligation of cell surface heparan sulfate proteoglycans by antibody-coated beads stimulates phagocytic uptake into epithelial cells: a model for cellular invasion by Neisseria gonorrhoeae. Exp Cell Res 1998;242:528-39.
38. Reis CR, Chen PH, Srinivasan S, Aguet F, Mettlen M, Schmid SL. Crosstalk between Akt/GSK3 $\beta$ signaling and dynamin-1 regulates clathrin-mediated endocytosis. EMBO J 2015;34: 2132-46.

39. van Deurs B, Röpke C, Thorball N. Kinetics of pinocytosis studied by flow cytometry. Eur J Cell Biol 1984;34:96-102.

40. Ishimoto H, Yanagihara K, Araki N, Mukae H, Sakamoto N, Izumikawa K, et al. Single-cell observation of phagocytosis by human blood dendritic cells. Jpn J Infect Dis 2008;61:294-7. 